\title{
Time Evolution of Two-Level Systems Driven by Periodic Fields
}

\author{
J. C. A. Barata and D. A. Cortez \\ Instituto de Física. Universidade de São Paulo \\ Caixa Postal 66 318. 05315970 São Paulo SP. Brasil \\ E-mail: jbarata@fma.if.usp.br and dacortez@fma.if.usp.br
}

\begin{abstract}
In this paper we study the time evolution of a class of two-level systems driven by periodic fields in terms of new convergent perturbative expansions for the associated propagator $U(t)$. The main virtue of these expansions is that they do not contain secular terms, leading to a very convenient method for quantitatively studying the long-time behaviour of that systems. We present a complete description of an algorithm to numerically compute the perturbative expansions. In particular, we applied the algorithm to study the case of an ac-dc field (monochromatic interaction), exploring various situations and showing results on (time-dependent) observable quantities, like transition probabilities. For a simple ac field, we analised particular situations where an approximate effect of dynamical localisation is exhibited by the driven system. The accuracy of our calculations was tested measuring the unitarity of the propagator $U(t)$, resulting in very small deviations, even for very long times compared to the cycle of the driving field.

PACS numbers: 03.65.-w, 02.30.Mv, 31.15.Md, 73.40.Gk
\end{abstract}

\section{INTRODUCTION}

Periodically (or more generically quasi-periodically) driven quantum two-level systems are of basic importance in many physical applications an have been widely studied since the pioneering works of Rabi [1], of Bloch and Siegert [2] and of Autler and Townes [3] (see also [4] for more recent discussions and [5, 6, 9] for other general references on the subject). The time evolution of such systems is governed by the Schrödinger equation (we henceforth adopt $\hbar=1$ )

$$
i \frac{d}{d t}|\Psi\rangle=H(t)|\Psi\rangle
$$

where $|\Psi\rangle=|\Psi(t)\rangle=\left(\begin{array}{c}\psi_{+}(t) \\ \psi_{-}(t)\end{array}\right)$ and $H(t)$ is the Hamiltonian of the system. We may consider, for instance, a spin$1 / 2$ system under the influence of a time-dependent (periodic) magnetic field $\vec{B}(t)$. In this situation, $H(t)$ takes the usual form $H(t)=-\frac{1}{2} \vec{B}(t) \cdot \vec{\sigma}$, where $\vec{\sigma}=\left(\sigma_{1}, \sigma_{2}, \sigma_{3}\right)$ are the Pauli matrices. The interest in the solution of (11) in this case is not restricted to the investigation of the quantum system. As first pointed by Feynman, Vernon and Hellwarth [10] (see also the discussion in [11]), the quantum system is equivalent to the classical Hamiltonian system describing a classical gyromagnet precessing in a magnetic field: $\frac{d}{d t} \overrightarrow{\mathcal{S}}=-\vec{B}(t) \times \overrightarrow{\mathcal{S}}$, where $\overrightarrow{\mathcal{S}}$ is a (three dimensional) unit vector. In fact, the methods described below can be directly applied to the analysis of this system as well, since the components of $\overrightarrow{\mathcal{S}}$ can be written in terms of the components $\psi_{ \pm}(t)$ of $|\Psi\rangle$ [10,11.

The evolution of the systems governed by (1) is determined by the time evolution operator $U(t, s)$ (also known as the propagator) which connects the state $|\Psi(s)\rangle$ at time $s$ to the state $|\Psi(t)\rangle$ at time $t:|\Psi(t)\rangle=$ $U(t, s)|\Psi(s)\rangle$. Defining $U(t)=U(t, 0)$ one has $U(t, s)=$
$U(t) U(s)^{\dagger}$. For a time-dependent Hamiltonian the propagator $U(t)$ can be computed via the Dyson expansion:

$$
U(t)=\hat{1}+\sum_{n=1}^{\infty}(-i)^{n} \int_{0}^{t} H\left(t_{1}\right) d t_{1} \cdots \int_{0}^{t_{n-1}} H\left(t_{n}\right) d t_{n} .
$$

Although (2) gives a straightforward manner to compute $U(t)$, the series in the r.h.s. is not generally uniformly convergent in time. For practical purposes this gives rise to difficulties when one is interested in the large-time behaviour of the system. For instance, if one considers a periodic Hamiltonian of the form $H(t)=\sum_{m} H_{m} e^{i m \omega t}$, with $H_{0} \neq 0$, two successive integrations in (2) would produce a linear term in $t$. Higher order terms in $t$ would appear with further integrations. These polynomial terms are known as secular terms and they plague the expansion of $U(t)$ in such a way that its uniform convergence is spoiled.

Of particular interest is the situation where the Schrödinger equation (11) takes the form

$$
i \frac{d}{d t}|\Psi\rangle=H_{1}(t)|\Psi\rangle, \quad \text { with } \quad H_{1}(t):=\epsilon \sigma_{3}-f(t) \sigma_{1},
$$

where $f(t)$ is a periodic function of time $t$ and $\epsilon$ is a real constant. By a rotation of $\pi / 2$ around the 2 -axis, we get the equivalent system

$$
i \frac{d}{d t}|\Phi\rangle=H_{2}(t)|\Phi\rangle, \quad \text { with } \quad H_{2}(t):=\epsilon \sigma_{1}+f(t) \sigma_{3},
$$

where $|\Phi\rangle=|\Phi(t)\rangle=\exp \left(-i \pi \sigma_{2} / 4\right)|\Psi(t)\rangle$. The Hamiltonian in (3) can be interpreted as describing a system with an unperturbed diagonal Hamiltonian $H_{0}:=\epsilon \sigma_{3}$, 
subjected to a (periodic) time-dependent perturbation $H_{I}(t):=-f(t) \sigma_{1}$, inducing a time-depending transition between the unperturbed eigenstates of $H_{0}$. Of course, (3) can be also interpreted as a spin- $1 / 2$ system under a magnetic field $\vec{B}(t)=(2 f(t), 0,-2 \epsilon)$.

In [6] - 8] we investigated the system described by (3) or (14) in the situation where $f$ is a periodic or quasi-periodic function of time and $\epsilon$ is "small" and a special perturbative expansion (power series in $\epsilon$ ) was developed, whose main virtue is to be free of secular terms. The algorithm employed involves an inductive "renormalisation" of a sort of effective field introduced through an exponential Ansatz (the function $g$, to be introduced below). Moreover, in the periodic case, we have been able in [7] to prove rigorously the uniform convergence of our expansions for all $t$, for $\epsilon$ sufficiently small, provided the function $f$ satisfies the special conditions I, II and III we describe below.

A feature of our method is the fact that we are able to present the complete (and convergent) $\epsilon$-expansion for the secular frequency $\Omega$ (also known as Rabi frequency) associated to the solution of (3)-(4). This is particularly important for the qualitative study of the large-time behaviour of that solutions. Another relevant conclusion is that, under conditions I-III, our method provides a complete perturbative solution of (3)-(4) for the monochromatic field (ad-dc field), except perhaps for spurious situations [8].

Since our series are uniformly convergent in time, it is possible to use them to study the long-time behaviour of observable quantities in a controlled way. It turns out that our expansions are also very practical for numerical calculations, providing very small errors even with few terms.

In this paper we describe the algorithm employed in the numerical computation of our perturbative solution and show the results obtained in the particular case of the monochromatic interactions. We begin in Section II by given a quick review of the perturbative method developed in 6 8]. Section III presents a description of the numerical algorithm developed to compute the expansions. Next, in Section IV, we show and discuss the results obtained in the case of monochromatic interactions. Finally, in Section $\mathrm{V}$, we draw some conclusions and final remarks.

\section{DESCRIPTION OF THE METHOD}

In [6], it was shown that the propagator $U(t)$ associated with the system (4) can be written as

$$
U(t)=\left(\begin{array}{cc}
R(t)\left(1+i g_{0} S(t)\right) & -i \epsilon R(t) S(t) \\
-i \epsilon \overline{R(t) S(t)} & \overline{R(t)}\left(1-i \overline{g_{0}} \overline{S(t)}\right)
\end{array}\right)
$$

where

$$
R(t):=\exp \left(-i \int_{0}^{t}(f(\tau)+g(\tau)) d \tau\right)
$$

and

$$
S(t):=\int_{0}^{t} R(\tau)^{-2} d \tau,
$$

where $g$ is a particular solution of the generalised Riccati equation

$$
g(t)^{\prime}-i g(t)^{2}-2 i f(t) g(t)+i \epsilon^{2}=0,
$$

and $g_{0} \equiv g(0)$. To solve (8) we pose

$$
g(t)=\sum_{n=1}^{\infty} G^{(n)}(t) \epsilon^{n}
$$

where $G^{(n)}(t):=q(t) c_{n}(t)$, with

$$
q(t):=\exp \left(i \int_{0}^{t} f(\tau) d \tau\right) .
$$

Inserting (9) into (8) yields a sequence of recursive equations for the coefficients $c_{n}$, whose solutions are

$$
\begin{aligned}
& c_{1}(t)=\alpha_{1} q(t) \\
& c_{2}(t)=q(t)\left[i \int_{0}^{t}\left(\alpha_{1}^{2} q(\tau)^{2}-q(\tau)^{-2}\right) d \tau+\alpha_{2}\right], \\
& c_{n}(t)=q(t)\left[i\left(\int_{0}^{t} \sum_{p=1}^{n-1} c_{p}(\tau) c_{n-p}(\tau) d \tau\right)+\alpha_{n}\right],
\end{aligned}
$$

for $n \geq 3$, where the $\alpha_{n}$ are arbitrary integration constants. Our strategy consists in fixing these constants inductively to cancel the secular terms. For instance, to cancel the secular term in $c_{2}$, the integrand in (11) cannot contain a constant term. Defining the mean value of a quasi-periodic function $h$ by

$$
M(h):=\lim _{T \rightarrow \infty} \frac{1}{2 T} \int_{-T}^{T} h(t) d t
$$

we must require that $M\left(\alpha_{1}^{2} q^{2}-q^{-2}\right)=0$. This leads to $\alpha_{1}^{2}=\overline{M\left(q^{2}\right)} / M\left(q^{2}\right)$, provided $M\left(q^{2}\right) \neq 0$. We remark that the mean value of a quasi-periodic function $h$ equals its zero order Fourier coefficient. In [6 8 we identified three classes of quasi-periodic functions $f$ for which this procedure of elimination of secular terms can be applied to all orders (i.e., to all functions $c_{n}$ ). These classes are defined by the following conditions:

$$
\begin{aligned}
\text { I. } & M\left(\mathcal{Q}_{0}\right) \neq 0, \\
\text { II. } & M\left(\mathcal{Q}_{0}\right)=0 \text { but } M\left(\mathcal{Q}_{1}\right) \neq 0, \\
\text { III. } & M\left(\mathcal{Q}_{0}\right)=M\left(\mathcal{Q}_{1}\right)=0 \text { but } M\left(\mathcal{Q}_{3}\right) \neq 0,
\end{aligned}
$$


where $\mathcal{Q}_{0}(t):=q(t)^{2}$,

$$
\begin{aligned}
& \mathcal{Q}_{1}(t):=\mathcal{Q}_{0}(t) \int_{0}^{t}\left(\mathcal{Q}_{0}(\tau)^{-1}-M\left(\mathcal{Q}_{0}^{-1}\right)\right) d \tau, \\
& \mathcal{Q}_{3}(t):=\mathcal{Q}_{0}(t) \int_{0}^{t}\left(\mathcal{Q}_{1}(\tau)-M\left(\mathcal{Q}_{1}\right)\right) d \tau .
\end{aligned}
$$

Under condition II, the Ansatz (9) has to be slightly modified to

$$
g(t)=\sum_{n=1}^{\infty} \widetilde{G}^{(n)}(t) \lambda^{n}, \quad \text { with } \quad \lambda:=\epsilon^{2}
$$

and $\widetilde{G}^{(n)}(t):=q(t) e_{n}(t)$. The solutions for $e_{n}$ are

$$
\begin{aligned}
& e_{1}(t)=q(t)\left(-i \int_{0}^{t} q(\tau)^{-2} d \tau+\beta_{1}\right) \\
& e_{n}(t)=q(t)\left[i\left(\int_{0}^{t} \sum_{p=1}^{n-1} e_{p}(\tau) e_{n-p}(\tau) d \tau\right)+\beta_{n}\right],
\end{aligned}
$$

for $n \geq 2$. Under condition III, we found in [8] the same solution for $g$ as in I, namely (9). The difference between the solutions obtained in I and III lie in the set of constants $\alpha_{n}$, which is conventionally fixed to remove the secular terms in each case.

In [7] we have shown that the $\epsilon$-expansion (9) or (15) converges uniformly for $|\epsilon|$ small and for all $t$, provided $f$ is periodic and satisfies I or II. In [8] we extended this result for case III. As we will discuss soon, case III is particularly important for the investigation of the dynamical localisation effect (also called, less properly, coherent destruction of tunnelling) for monochromatic interactions.

The secular frequency $\Omega$, in conditions I or III, is given by [月] 9

$$
\Omega=M(f)+M(g)=F_{0}+\sum_{n=1}^{\infty} M\left(G^{(n)}\right) \epsilon^{n}
$$

and, in condition II, by

$$
\Omega=F_{0}+\sum_{n=1}^{\infty} M\left(\widetilde{G}^{(n)}\right) \lambda^{n} .
$$

Hence, with our previous definitions, for condition I, we have

$$
\begin{aligned}
\Omega & =F_{0}+\epsilon \alpha_{1} M\left(\mathcal{Q}_{0}\right) \\
& +\epsilon^{2}\left[i \alpha_{1}^{2} M\left(\mathcal{Q}_{2}\right)-i M\left(\mathcal{Q}_{1}\right)+\alpha_{2} M\left(\mathcal{Q}_{0}\right)\right] \\
& +\epsilon^{3}\left[2 \alpha_{1} M\left(\mathcal{Q}_{3}\right)+\alpha_{3} M\left(\mathcal{Q}_{0}\right)\right]+\mathcal{O}\left(\epsilon^{4}\right),
\end{aligned}
$$

where

$$
\mathcal{Q}_{2}(t):=\mathcal{Q}_{0}(t) \int_{0}^{t}\left(\mathcal{Q}_{0}(\tau)-M\left(\mathcal{Q}_{0}\right)\right) d \tau .
$$

One easily sees, by computing the zero order Fourier coefficient of $\mathcal{Q}_{2}$ (details in $[8]$ ), that $M\left(\mathcal{Q}_{2}\right)=0$ whenever $M\left(\mathcal{Q}_{0}\right)=0$. Hence, for condition II, we get

$$
\Omega=F_{0}-i \epsilon^{2} M\left(\mathcal{Q}_{1}\right)+\mathcal{O}\left(\epsilon^{4}\right)
$$

and, for condition III,

$$
\Omega=F_{0}+2 \alpha_{1} \epsilon^{3} M\left(\mathcal{Q}_{3}\right)+\mathcal{O}\left(\epsilon^{4}\right) .
$$

\section{NUMERICAL COMPUTATION OF THE SOLUTION}

We present here a step-by-step algorithm which enables us to compute the propagator (5) solely from the Fourier coefficients of the (periodic) interaction $f$. This algorithm is based on the convergent expansions presented in [7], which expresses the propagator $U(t)$ in terms of its Floquet form.

Let us first begin with some conventions. We suppose that the interaction function $f$ is periodic with period $T_{\omega}=2 \pi / \omega$ and that its Fourier decomposition $f=\sum_{n} F_{n} e^{i n \omega t}$ has only a finite number of terms. Since $f$ is real, excluding the constant term $F_{0}, f$ must have an even number of non-vanishing Fourier coefficients, say $2 J$, with $J \geq 1$. Denoting the set of integers $\left\{n \in \mathbb{Z}, n \neq 0 \mid F_{n} \neq 0\right\}$ by $\left\{n_{1}, \ldots, n_{2 J}\right\}$, we may write

$$
f(t)=F_{0}+\sum_{a=1}^{2 J} f_{a} e^{i n_{a} \omega t},
$$

with the convention that $n_{a}=-n_{2 J-a+1}$, for all $1 \leq a \leq$ $J$, and with $f_{a} \equiv F_{n_{a}}$. Clearly $\overline{f_{a}}=f_{2 J-a+1}, 1 \leq a \leq J$.

\section{A. Interactions with $F_{0}=0$}

In this case, the Fourier decomposition of the functions $q$ and $q^{2}$ can be written as [6,7]

$$
q(t)=\sum_{m \in \mathbb{Z}} Q_{m} e^{i m \omega t} \text { and } q(t)^{2}=\sum_{m \in \mathbb{Z}} Q_{m}^{(2)} e^{i m \omega t} .
$$

The coefficients $Q_{m}$ and $Q_{m}^{(2)}$ are of basic importance in the numerical computation of the propagator (5). They can be obtained in a closed form from the coefficients $F_{m}$ of $f$. Explicitly, we have [6.7]

$$
Q_{m}=e^{i \gamma_{f}} \sum_{p_{1}, \ldots, p_{2 J}=0}^{\infty} \delta(P, m) \prod_{a=1}^{2 J}\left[\frac{1}{p_{a} !}\left(\frac{f_{a}}{n_{a} \omega}\right)^{p_{a}}\right],
$$

for all $m$, where

$$
P \equiv P\left(p_{1}, \ldots, p_{2 J}, n_{1}, \ldots, n_{2 J}\right):=\sum_{b=1}^{2 J} p_{b} n_{b},
$$


and

$$
\gamma_{f}:=i \sum_{a=1}^{2 J} \frac{f_{a}}{n_{a} \omega} .
$$

The symbol $\delta(P, m)$ denotes the Krönecker delta: $\delta(P, m)=1$, if $P=m$ and $\delta(P, m)=0$, otherwise. To compute the Fourier coefficients of $q^{2}$, we simply note that $q^{2}$ is obtained from $q$ by the substitution $f \rightarrow 2 f$. Hence, for all $m$,

$$
Q_{m}^{(2)}=e^{2 i \gamma_{f}} \sum_{p_{1}, \ldots, p_{2 J}=0}^{\infty} \delta(P, m) \prod_{a=1}^{2 J}\left[\frac{1}{p_{a} !}\left(\frac{2 f_{a}}{n_{a} \omega}\right)^{p_{a}}\right] .
$$

Formulas (22) and (23) can be computed either analytically (in some cases) or numerically. For the monochromatic interactions, a closed form in terms of Bessel functions of first kind is obtained. The exact result will be presented in Section IV.

Once we know the coefficients $Q_{m}$ and $Q_{m}^{(2)}$, we proceed to compute the particular solution (9) or (15) of the generalised Riccati equation (8). To decide whether we use (9) or (15), we must check which condition I, II or III is satisfied by $f$. Hence, we have to look at the mean values of $\mathcal{Q}_{0}, \mathcal{Q}_{1}$ and $\mathcal{Q}_{3}$. One obviously has $M\left(\mathcal{Q}_{0}\right)=M\left(q^{2}\right)=Q_{0}^{(2)}$. To obtain $M\left(\mathcal{Q}_{1}\right)$ and $M\left(\mathcal{Q}_{3}\right)$, we need the Fourier decompositions of $\mathcal{Q}_{1}$ and $\mathcal{Q}_{3}$. From the definitions (13) and (14), after some simple computations, we get

$$
M\left(\mathcal{Q}_{1}\right)=\frac{i}{\omega} \sum_{\substack{m \in \mathbb{Z} \\ m \neq 0}} \frac{\overline{Q_{-m}^{(2)}}\left(Q_{0}^{(2)}-Q_{-m}^{(2)}\right)}{m}
$$

and

$$
\begin{aligned}
M\left(\mathcal{Q}_{3}\right) & =-\frac{1}{\omega^{2}} \sum_{\substack{n, m \in \mathbb{Z} \\
n \neq 0, m \neq 0}} \frac{\overline{Q_{-m}^{(2)}}}{n m}\left(Q_{0}^{(2)} Q_{n}^{(2)}\right. \\
& \left.-Q_{0}^{(2)} Q_{n-m}^{(2)}+Q_{-n}^{(2)} Q_{n-m}^{(2)}\right) .
\end{aligned}
$$

The numerical value of $M\left(\mathcal{Q}_{1}\right)$ and $M\left(\mathcal{Q}_{3}\right)$ can be calculated trivially from the above expressions once $Q_{m}^{(2)}$ are known.

\section{Computing $g(t)$ in Cases I and III}

We remember that condition I applies whenever $f$ satisfies $M\left(\mathcal{Q}_{0}\right) \neq 0$. In this case, by properly fixing the constants $\alpha_{n}$, we can completely eliminate the secular terms from the functions $c_{n}$ [6.6]. For this reason, we may write

$$
c_{n}(t)=\sum_{m \in \mathbb{Z}} C_{m}^{(n)} e^{i m \omega t}
$$

The Fourier coefficients $C_{m}^{(n)}$ are obtained from equations (10)-(12). Their inductive structure is given by the relations [6]

$$
\begin{aligned}
C_{m}^{(1)} & =\alpha_{1} Q_{m}, \\
C_{m}^{(2)} & =\sum_{\substack{n_{1} \in \mathbb{Z} \\
n_{1} \neq 0}} \frac{Q_{m-n_{1}}\left(\alpha_{1}^{2} Q_{n_{1}}^{(2)}-\overline{Q_{-n_{1}}^{(2)}}\right)}{n_{1} \omega}+\alpha_{2} Q_{m}, \\
C_{m}^{(n)} & =\sum_{\substack{n_{1}, n_{2} \in \mathbb{Z} \\
n_{1}+n_{2} \neq 0}} \frac{Q_{m-\left(n_{1}+n_{2}\right)}}{\left(n_{1}+n_{2}\right) \omega}\left(\sum_{p=1}^{n-1} C_{n_{1}}^{(p)} C_{n_{2}}^{(n-p)}\right) \\
& +\alpha_{n} Q_{m}, \text { for } n \geq 3 .
\end{aligned}
$$

The constants $\alpha_{n}$ have closed forms in terms of the coefficients $Q_{m}^{(2)}$ and $C_{m}^{(p)}$, for $p \leq n-1$. Since they involve somewhat large expressions, we refrain from writing them here. The complete expressions can be found in [6]. We may see that the whole inductive structure of the coefficients $C_{m}^{(n)}$ is known and, therefore, the computation of $c_{n}$ is just a matter of numerically evaluating the above expressions.

Since in case I $g$ is given by (9), we may write

$$
g(t)=q(t) \sum_{n=1}^{\infty} c_{n}(t) \epsilon^{n}=: \sum_{n=1}^{\infty}\left(\sum_{m \in \mathbb{Z}} G_{m}^{(n)} e^{i m \omega t}\right) \epsilon^{n},
$$

where $G_{m}^{(n)}$ is given by the convolution

$$
G_{m}^{(n)}=\sum_{p \in \mathbb{Z}} Q_{m-p} C_{p}^{(n)},
$$

whose numerical value can be easily computed since we already know $Q_{m}$ and $C_{m}^{(n)}$, for all $m$ and $n$. This gives $g$ for all $t$ under condition I. For condition III $\left(M\left(\mathcal{Q}_{0}\right)=M\left(\mathcal{Q}_{1}\right)=0\right.$, but $\left.M\left(\mathcal{Q}_{3}\right) \neq 0\right)$ one has essentially the same solution except for the constants $\alpha_{n}$ which are calculated differently from condition I. Their formulas can be found in [8].

\section{Computing $g(t)$ in Case $I I$}

Condition II applies whenever $f$ satisfies $M\left(\mathcal{Q}_{0}\right)=0$, but $M\left(\mathcal{Q}_{1}\right) \neq 0$. In this case, the perturbative solution of the generalised Riccati equation (8) is given by (15). The constants $\beta_{n}$ which appear in the functions $e_{n}$ (equations (16)-(17)) can be chosen such that no secular terms emerge [6]. Hence, with this particular choice of the constants $\beta_{n}$, we may write

$$
e_{n}(t)=\sum_{m \in \mathbb{Z}} E_{m}^{(n)} e^{i m \omega t}
$$

The recursive structure of the coefficients $E_{m}^{(n)}$ is given by [6] 


$$
\begin{aligned}
E_{m}^{(1)} & =-\sum_{\substack{n \in \mathbb{Z} \\
n \neq 0}} \frac{Q_{m-n} \overline{Q_{-n}^{(2)}}}{n \omega}+Q_{m}\left(\beta_{1}+\sum_{\substack{n \in \mathbb{Z} \\
n \neq 0}} \frac{\overline{Q_{-n}^{(2)}}}{n \omega}\right), \\
E_{m}^{(n)} & =\sum_{\substack{n_{1}, n_{2} \in \mathbb{Z} \\
n_{1}+n_{2} \neq 0}} \frac{Q_{m-n_{1}-n_{2}}}{\left(n_{1}+n_{2}\right) \omega}\left(\sum_{p=1}^{n-1} E_{n_{1}}^{(p)} E_{n_{2}}^{(n-p)}\right) \\
& -Q_{m} \sum_{\substack{n_{1}, n_{2} \in \mathbb{Z} \\
n_{1}+n_{2} \neq 0}}\left(\sum_{p=1}^{n-1} E_{n_{1}}^{(p)} E_{n_{2}}^{(n-p)}\right) \frac{1}{\left(n_{1}+n_{2}\right) \omega} \\
& +\beta_{n} Q_{m}, \quad \text { for } n \geq 2 .
\end{aligned}
$$

The constants $\beta_{n}$ have also closed forms [6] in terms of the known $Q_{m}^{(2)}$ and $E_{m}^{(p)}$, for all $m$ and $p \leq n-1$. Thus, the solution (15) can be evaluated numerically in any order for all $t$. For future convenience, we write

$$
g(t)=q(t) \sum_{n=1}^{\infty} e_{n}(t) \lambda^{n}=: \sum_{n=1}^{\infty}\left(\sum_{m \in \mathbb{Z}} \widetilde{G}_{m}^{(n)} e^{i m \omega t}\right) \lambda^{n},
$$

where $\widetilde{G}_{m}^{(n)}$ is given by

$$
\widetilde{G}_{m}^{(n)}=\sum_{p \in \mathbb{Z}} Q_{m-p} E_{p}^{(n)},
$$

which completely specifies $g$ for all $t$ under condition II.

\section{Computing the Propagator in Cases I, II and III}

Once the coefficients $G_{m}^{(n)}$ (for cases I and III) or $\widetilde{G}_{m}^{(n)}$ (for case II) are known, the propagator $U(t)$ expressed in (5) can be computed in a straightforward manner, as will be shown now. We illustrate our procedure with the coefficients $G_{m}^{(n)}$ of conditions I and III. For condition II, where the coefficients of $g$ are $\widetilde{G}_{m}^{(n)}$, the discussed procedure has to be adapted with self-evident modifications.

We begin by defining

$$
G_{m}(\epsilon):=\sum_{n=1}^{\infty} G_{m}^{(n)} \epsilon^{n} .
$$

The secular frequency (18) is clearly given by (see 24)

$$
\Omega \equiv \Omega(\epsilon)=\sum_{n=1}^{\infty} G_{0}^{(n)} \epsilon^{n},
$$

since we are supposing that $F_{0}=0$. Hence, by (24),

$$
g(t)=\Omega+\sum_{\substack{m \in \mathbb{Z} \\ m \neq 0}} G_{m}(\epsilon) e^{i m \omega t} .
$$

Looking at expression (5) for the propagator, we see that the Fourier series of $R$ (see (6)) can be computed if we first find the Fourier decomposition of

$$
W(t):=\exp \left(-i \int_{0}^{t} g(\tau) d \tau\right) .
$$

Indeed, since $R=\bar{q} W$, we obtain the Fourier series of $R$ by taking a convolution of the coefficients of $\bar{q}$ and $W$.

It is easy to see that

$$
W(t)=e^{i \gamma_{f}(\epsilon)} e^{-i \Omega t} \exp \left(-\sum_{m \in \mathbb{Z}} H_{m} e^{i m \omega t}\right),
$$

with

$$
H_{m} \equiv H_{m}(\epsilon):=\frac{G_{m}(\epsilon)}{m \omega}, \quad \text { for } m \neq 0
$$

and $H_{0}=0$. Moreover,

$$
\gamma_{f}(\epsilon):=i \sum_{m \in \mathbb{Z}} H_{m} .
$$

Writing

$$
W(t)=e^{-i \Omega t} \sum_{m \in \mathbb{Z}} W_{m} e^{i m \omega t},
$$

and using (28), we find

$$
\begin{gathered}
W_{m} \equiv W_{m}(\epsilon)=e^{-i \gamma_{f}(\epsilon)}\left(-H_{m}+\sum_{p=1}^{\infty} \frac{(-1)^{p+1}}{(p+1) !}\right. \\
\left.\times \sum_{n_{1}, \ldots, n_{p} \in \mathbb{Z}} H_{n_{1}} \cdots H_{n_{p}} H_{m-N_{p}}\right), \quad \text { for } m \neq 0
\end{gathered}
$$

and

$$
\begin{array}{r}
W_{0} \equiv W_{0}(\epsilon)=e^{-i \gamma_{f}(\epsilon)}\left(1+\sum_{p=1}^{\infty} \frac{(-1)^{p+1}}{(p+1) !}\right. \\
\left.\times \sum_{n_{1}, \ldots, n_{p} \in \mathbb{Z}} H_{n_{1}} \cdots H_{n_{p}} H_{-N_{p}}\right),
\end{array}
$$

with $N_{p}:=\sum_{a=1}^{p} n_{a}$. Using now (29) and the fact that $R=\bar{q} W$, we conclude that $R$ can be written as

$$
R(t)=e^{-i \Omega t} \sum_{m \in \mathbb{Z}} R_{m} e^{i m \omega t},
$$

with the coefficients $R_{m}$ given by the convolution

$$
R_{m}=\sum_{p \in \mathbb{Z}} \overline{Q_{p-m}} W_{p}
$$

This finishes with the computation of $R$ in terms of its Fourier series (30). We note from formulas (26)-(31) that once the coefficients $G_{m}^{(n)}$ of $g$ are given, we can numerically evaluate $G_{m}, H_{m}, W_{m}$ and, hence, $R_{m}$ with a trivial computer code. 
Next we proceed to compute the Fourier series of $S$ (see (7)). First we find the Fourier coefficients of $R^{-2}$. This is an easy task since $R^{-2}$ is obtained from $R$ by replacing $(f+g) \rightarrow-2(f+g)$. Hence, we must replace $H_{m} \rightarrow-2 H_{m}$ and $\bar{q} \rightarrow q^{2}$. Consequently, we get

$$
R(t)^{-2}=e^{2 i \Omega t} \sum_{m \in \mathbb{Z}} R_{m}^{(-2)} e^{i m \omega t},
$$

where

$$
R_{m}^{(-2)}=\sum_{p \in \mathbb{Z}} Q_{m-p}^{(2)} W_{p}^{(-2)}
$$

with

$$
\begin{gathered}
W_{m}^{(-2)} \equiv W_{m}^{(-2)}(\epsilon)=e^{2 i \gamma_{f}(\epsilon)}\left(2 H_{m}+\sum_{p=1}^{\infty} \frac{2^{p+1}}{(p+1) !}\right. \\
\left.\quad \times \sum_{n_{1}, \ldots, n_{p} \in \mathbb{Z}} H_{n_{1}} \cdots H_{n_{p}} H_{m-N_{p}}\right), \text { for } m \neq 0
\end{gathered}
$$

and

$$
\begin{array}{r}
W_{0}^{(-2)} \equiv W_{0}^{(-2)}(\epsilon)=e^{2 i \gamma_{f}(\epsilon)}\left(1+\sum_{p=1}^{\infty} \frac{2^{p+1}}{(p+1) !}\right. \\
\left.\times \sum_{n_{1}, \ldots, n_{p} \in \mathbb{Z}} H_{n_{1}} \cdots H_{n_{p}} H_{-N_{p}}\right) .
\end{array}
$$

Now $S$ is obtained by a simple integration of $R^{-2}$. A trivial computation from (32), gives

$$
S(t)=\sigma_{0}+e^{2 i \Omega t} \sum_{m \in \mathbb{Z}} S_{m} e^{i m \omega t},
$$

with

$$
S_{m}:=-i \frac{R_{m}^{(-2)}}{m \omega+2 \Omega} \quad \text { and } \quad \sigma_{0}:=-\sum_{m \in \mathbb{Z}} S_{m} .
$$

We assume that $m \omega+2 \Omega \neq 0$ for all $m \in \mathbb{Z}$ (see the discussion of crossings in 77).

We have found expressions for $R$ and $S$ in terms of its Fourier series. This series converge absolutely and uniformly as we showed in [7, 8]. To compute the propagator given in (5), we still need $g_{0}$, which can be easily obtained from 27$)$ :

$$
g_{0} \equiv g(0)=\Omega+\sum_{\substack{m \in \mathbb{Z} \\ m \neq 0}} G_{m}(\epsilon)
$$

Formulas (30), (33) and (35) can now be used to evaluate $U(t)$ for all times. We stress that these formulas depend essentially on the Fourier coefficients $G_{m}^{(n)}$ of $g$. These, in turn, depend on $C_{m}^{(n)}$ which are direct linked to $Q_{m}$ and $Q_{m}^{(2)}$, derived from the Fourier coefficients of the interaction function $f$. In short, to help us visualise the necessary steps towards the computation of $U(t)$, we may draw the following "chain":

$$
\begin{array}{cccc}
f(t) & \rightarrow F_{m} \rightarrow Q_{m}, Q_{m}^{(2)} \rightarrow \alpha_{n} \\
\downarrow & \quad \leftarrow G_{m}^{(n)}, g_{0} \leftarrow C_{m}^{(n)} \\
H_{m} & \leftarrow \quad \\
\downarrow & & \\
W_{m}, W_{m}^{(-2)} & \rightarrow R_{m}, S_{m} \rightarrow R(t), S(t) \rightarrow U(t) .
\end{array}
$$

\section{B. Interactions with $F_{0} \neq 0$}

When $F_{0} \neq 0$, one automatically has $M\left(\mathcal{Q}_{0}\right)=0$, except perhaps when $2 F_{0}=k \omega$, for some integer $k$. These facts were shown in [6,7]. Since the situation where $2 F_{0}=k \omega$, for some integer $k$, was nowhere investigated in our previous works 6 [ 80, we will ignore this possibility by henceforth assuming that $2 F_{0} \neq k \omega$, for all $k \in \mathbb{Z}$. Since $M\left(\mathcal{Q}_{0}\right)=0$, condition I is never satisfied when $F_{0} \neq 0$. The convergence of our expansions for $F_{0} \neq 0$ in condition III, however, has not yet been studied. Hence, we will only consider condition II. In this case [7], the function $q$ turns to be

$$
q(t)=e^{i F_{0} t} \sum_{m \in \mathbb{Z}} Q_{m} e^{i m \omega t}
$$

and the functions $e_{n}$ (see (16)-(17)),

$$
e_{n}(t)=e^{-i F_{0} t} \sum_{m \in \mathbb{Z}} E_{m}^{(n)} e^{i m \omega t} \text {. }
$$

When $F_{0} \neq 0$, the coefficients $E_{m}^{(n)}$ in the above expression assume a simple form [7]:

$$
\begin{aligned}
E_{m}^{(1)} & =\sum_{a \in \mathbb{Z}} \frac{Q_{m+a} \overline{Q_{a}^{(2)}}}{a \omega+2 F_{0}}, \\
E_{m}^{(n)} & =\sum_{p=1}^{n-1} \sum_{a, b \in \mathbb{Z}} \frac{Q_{m-a-b} E_{a}^{(p)} E_{b}^{(n-p)}}{(a+b) \omega-2 F_{0}}, \quad \text { for } n \geq 2 .
\end{aligned}
$$

Finally, from (36) and (37), we conclude that $g$ given in (25) assumes the form

$$
g(t)=\sum_{m \in \mathbb{Z}} \widetilde{G}_{m} e^{i m \omega t}
$$

where

$$
\widetilde{G}_{m} \equiv \widetilde{G}_{m}(\epsilon)=\sum_{n=1}^{\infty} \widetilde{G}_{m}^{(n)} \lambda^{n}
$$


with $\lambda=\epsilon^{2}$ and

$$
\widetilde{G}_{m}^{(n)}=\sum_{p \in \mathbb{Z}} Q_{m-p} E_{p}^{(n)}
$$

This gives $g$ in terms of its Fourier series.

To compute the propagator $U(t)$ accordantly to (5), all we have to do is follow the procedure detailed in Section III A 3 with the replacement of $G_{m}^{(n)} \rightarrow \widetilde{G}_{m}^{(n)}$ and of $\epsilon \rightarrow \lambda$.

\section{NUMERICAL RESULTS FOR THE MONOCHROMATIC INTERACTIONS}

We now apply the algorithm discussed in Section [II] to study the system (3)-(14) under the influence of monochromatic interactions (ac-dc field):

$$
f(t)=F_{0}+\varphi \cos (\omega t),
$$

which are relevance for many physical applications. With the conventions introduced in Section [II], we have $J=1$, $f_{1}=f_{2}=\varphi / 2, n_{1}=-n_{2}=-1$. A simple application of formula (23) gives

$$
\mathcal{Q}_{0}(t)=\sum_{n \in \mathbb{Z}} J_{n}\left(\chi_{1}\right) e^{i\left(n+\chi_{2}\right) \omega t},
$$

where $J_{n}$ is the Bessel function of first kind and order $n$ and where we defined $\chi_{1}:=2 \varphi / \omega$ and $\chi_{2}:=2 F_{0} / \omega$. Depending on the parameters $\chi_{1}$ and $\chi_{2}$, the function $f$ given in (38) satisfies one of conditions I, II or III, except, perhaps, for spurious situations. The detailed analysis of these facts can be found in [8]. Table [1] summarises the conclusions presented in [8] and gives a classification of the conditions satisfied by $f$ as a function of the parameters $\chi_{1}$ and $\chi_{2}$.

\begin{tabular}{lccc}
\hline \hline Label & $\chi_{1}$ & $\chi_{2}$ & Condition \\
\hline (A) & not a zero of $J_{m}$ & $-m \in \mathbb{Z}$ & I \\
$(\mathrm{B})$ & zero of $J_{m}$ & $-m \in \mathbb{Z}$ & III \\
(C) & any & not integer & II \\
\hline \hline
\end{tabular}

TABLE I. Classification of the conditions satisfied by the monochromatic interactions $f(t)=F_{0}+\varphi \cos (\omega t)$ as a function of the parameters $\chi_{1}=2 \varphi / \omega$ and $\chi_{2}=2 F_{0} / \omega$. We labelled by (A), (B) and (C) the three possible cases.

Although not indicated in Table If there is in case (C), on each interval $(k, k+1), k=1,2, \ldots$, a special value $\chi_{2}^{s}$ of $\chi_{2}$ (depending on $\chi_{1}$ ) for which $M\left(\mathcal{Q}_{1}\right)=0$, and we would be out of condition II 8 . We refrain from studying this rather spurious situation here. A more detailed analysis of this case can be found in [8].
We next show some graphical results of transition probabilities calculated via the algorithm described in section III for the situations (A), (B) and (C) described in Table [1. Let $\left|\Phi_{+}\right\rangle=\left(\begin{array}{l}1 \\ 0\end{array}\right)$ and $\left|\Phi_{-}\right\rangle=\left(\begin{array}{l}0 \\ 1\end{array}\right)$ be two orthogonal states of a system described by (14) (the eigenstates of the unperturbed Hamiltonian $H_{0}$ of $H_{1}$ in (3)). The probability for the transition from the initial state $\left|\Phi_{+}\right\rangle$to the final state $\left|\Phi_{-}\right\rangle$at time $t$ is given by

$$
P(t):=\left|\left\langle\Phi_{+}|U(t)| \Phi_{-}\right\rangle\right|^{2}=\left|U_{12}(t)\right|^{2} .
$$

We can evaluate $P(t)$ numerically using the methods described in Section III to compute $U(t)$. To estimate the accuracy of our calculations, we tested the unitarity of the time evolution operator, $U(t)^{\dagger} U(t)=\hat{1}$, and considered the quantity

$$
N(t):=\left|U_{11}(t)\right|^{2}+\left|U_{12}(t)\right|^{2}-1,
$$

which should be identically equal to 0 for unitary $U(t)$.

Let us first consider case (A) of Table fi with $\omega=1.0$, $\chi_{1}=2 / \omega$ and $\chi_{2}=F_{0}=0$. Figure 11 shows graphs of $P(t)$ and $N(t)$ for $\epsilon=0.01, \epsilon=0.10$ and $\epsilon=0.40$, plotted from $t=0$ to $t=T_{\Omega}=2 \pi / \Omega$, in units of $T_{\omega}=2 \pi / \omega$, the cycle of the external field. The calculations were performed using an expansion for $g$ up to $\mathcal{O}\left(\epsilon^{25}\right)$. We took all the Fourier coefficients (generically called $\mathcal{F}_{m}$ ) involved in the computations of $U(t)$ within the range $m=-40, \ldots, 40$. From the deviations of $N(t)$ from 0 , we can infer very small errors in the calculations, leading to very accurate values of $P(t)$. For $\epsilon=0.01$ and $\epsilon=0.10$, we have errors of the order of only $4.0 \times 10^{-5} \%$. For $\epsilon=0.40$, the errors jump to $6.0 \times 10^{-2} \%$, indicating that the parameter $\epsilon$ is coming close to the radius of convergence of our expansions. Since in case (A) we are under condition I, the secular frequency $\Omega$ is given by (19), hence $\Omega=\mathcal{O}(\epsilon)$ (we choose $F_{0}=\chi_{2}=0$ ). For $\epsilon=0.01$, from the full expansion of $\Omega$ (see (18)), we get $T_{\Omega} \cong 450 T_{\omega}$. For $\epsilon=0.10, T_{\Omega} \cong 45 T_{\omega}$ and for $\epsilon=0.40$, $T_{\Omega} \cong 25 T_{\omega}$. We may notice from the graphs of Figure 1, that the transition probability $P(t)$ behaves like a Rabi oscillation with a frequency $\Omega$, i.e. $P(t) \cong \sin ^{2}(\Omega t)$. Small oscillations of frequency $\omega$, with amplitudes of or$\operatorname{der}|\epsilon|$, are superposed with this Rabi oscillation, leading to a quasi-periodic evolution for the system (as ensured by the Floquet theorem).

We now consider case (B) of Table with $\omega=10.0$, $\chi_{1}=x_{1}, x_{1}$ being the first positive zero of $J_{0}$, and $\chi_{2}=F_{0}=0$. Since we are under condition III and $F_{0}=0$ the secular frequency becomes, according to (21), $\Omega=\mathcal{O}\left(\epsilon^{3}\right)$, a fact first pointed in [9]. This weak dependence on $\epsilon$ indicates long transition times for the probability amplitude $P(t)$. This phenomenon is known as the dynamical localisation effect [12] (see [5] for a general criterion and for a more complete list of references). To compute the solution in this case, $g$ was expanded up to $\epsilon^{6}$. This contrasts with the expansion for $g$ in the previous situation, where $g$ has been computed up to $\mathcal{O}\left(\epsilon^{25}\right)$. 
The reason for this lies in the fact that under condition III the expressions for the constants $\alpha_{n}$ (see (10)-(12)) are somewhat more intricate than those of condition I. Fortunately, however, the expressions for the constants $\alpha_{1}, \alpha_{2}$ and $\alpha_{3}$ are not so complicated and this suffices to give an expansion of $g$ free of secular terms up to the $\epsilon^{6}$ term (see [8] for details). If we limit ourself to study situations of small $\epsilon$, i.e. well inside of the radius of convergence of our expansions, excellent results can be achieved. To improve the accuracy of our results, it is better to use a larger value of $\omega$.

Figure 2 shows graphs of the numerical values of $P(t)$ and $N(t)$ for $\epsilon=0.01, \epsilon=0.10$ and $\epsilon=0.20$, plotted from $t=0$ to $t=T_{\Omega}=2 \pi / \Omega$, in units of $T_{\omega}=2 \pi / \omega$. The errors in Figure 2 (measured out of the deviations of $N(t)$ from zero) are greater than those presented in Figure 1 due to the lower order $\epsilon$-expansion of $g$ in the former case. However, for small $\epsilon$, the errors are still small, being of the order of only $3.0 \times 10^{-3} \%$ for $\epsilon=0.01$ and of the order of $3.0 \times 10^{-1} \%$ for $\epsilon=0.10$. For larger $\epsilon(=0.20)$, the errors reach the value of about $1.0 \%$, indicating that a higher order $\epsilon$-expansion is needed to improve accuracy. We observe that the plots of $P(t)$ in Figure 2 have the same qualitative aspect of those in Figure 11. There is a predominant Rabi oscillation (with frequency $\Omega$ ) aspect superposed by minor oscillations of frequency $\omega$ whose amplitudes are possible bounded by $|\epsilon|^{3}$ terms. This fact, however, has yet not been proven directly from our expansions, so it has to be faced more as a qualitative analysis rather than a rigorous quantitative one.

The main distinction between the graphs of Figure 1 and those of Figure 2 is, undoubtedly, the Rabi oscillation period $T_{\Omega}$. In the later, the long time needed for the system to transit from the initial state $\left|\Phi_{+}\right\rangle$to the final state $\left|\Phi_{-}\right\rangle$, compared to the basic cycle $T_{\omega}$ of the external perturbation, is the (approximate) dynamical localisation effect. The secular period $T_{\Omega}$ obtained for the situations studied in Figure 2 were $T_{\Omega} \cong 1.6 \times 10^{9} T_{\omega}$ for $\epsilon=0.01$, $T_{\Omega} \cong 1.6 \times 10^{6} T_{\omega}$ for $\epsilon=0.10$ and $T_{\Omega} \cong 2.2 \times 10^{5} T_{\omega}$ for $\epsilon=0.20$. These are somewhat much larger values of $T_{\Omega}$ than those of Figure 1.

As we have mentioned, the graphs of Figure 2 were computed with $\chi_{1}=x_{1}$, where $x_{1}$ is the first positive zero of $J_{0}$. It is interesting to test our solution considering other possible zeros of $J_{0}$. In Figure 3 we show plots of $P(t)$ and $N(t)$ calculated with $\chi_{2}=x_{2}$, the second positive zero of $J_{0}$ (we also took $\omega=10.0$ and, of course, $\left.\chi_{2}=0\right)$. The three situations presented in Figure 3 correspond to $\epsilon=0.10, \epsilon=0.20$ and $\epsilon=0.30$. We may note that the qualitative behaviour of $P(t)$ presented in Figure 3 is the same of Figure 2. In particular, the effect of dynamical localisation is preserved since we still have $\Omega=\mathcal{O}\left(\epsilon^{3}\right)$ when $\chi_{1}=x_{2}$. We may note from the deviation of unitarity $N(t)$ shown in Figure 3 that the errors, compared with the ones in Figure 2 for the same values of $\epsilon$, are smaller by a factor $\sim 1 / 10$. We may understand this fact as follows: since $x_{2}>x_{1}$, the strength $\varphi$ of the interaction presented in Figure 3 is greater than in Figure 2, hence, for the same values of $\epsilon$, the effective perturbation $\epsilon / \varphi$ is smaller in the former case, leading to a more precise perturbative computation.

We now investigate case $(\mathrm{C})$ of Table I. We consider $\omega=1.0, \chi_{1}=1.0$ and $\chi_{2}=0.3$. This implies condition II and we have to follow the prescriptions of Section IIIB. Figure 4 shows the results obtained for $P(t)$ and $N(t)$ for three values of $\epsilon$ : $0.05,0.10$ and 0.20 . The various plots were calculated using an expansion of $g$ up to $\epsilon^{20}$. It was not necessary, thus, to use large $\omega$ to ensure convergence of the expansions, as we did in the situations presented in Figure 2 (condition III). As usual, $P(t)$ and $N(t)$ were computed from $t=0$ to $t=T_{\Omega}$ in units of $T_{\omega}$. The qualitative behaviour of the transition probability is significantly different from the previous results (Figures 1 and 2). This is a consequence of the non-vanishing constant field $F_{0}$ presented in the interaction $f$. Indeed, the secular frequency $\Omega$ is now given by (20) and, hence, is of order of $F_{0}$. For $\epsilon=0.05$, we obtained $T_{\Omega} \cong 6.3 T_{\omega}$, for $\epsilon=0.15, T_{\Omega} \cong 6.0 T_{\omega}$ and for $\epsilon=0.20, T_{\Omega} \cong 5.0 T_{\omega}$. Since we choose $F_{0}$ as the same order of $\omega$, there is a strong competition between the Rabi oscillation (governed by $\Omega$ ) and the external field oscillations. This leads to the patterns shown in Figure 1 , which do not behave purely like $\sin ^{2}(\Omega t)$. In particular, $P(t)<1$ for all times, leading to the conclusion that the state $\left|\Phi_{+}\right\rangle$never transits completely to $\left|\Phi_{-}\right\rangle$. One sees, moreover, that the transition amplitude $P(t)$ tends to zero as $\epsilon \rightarrow 0$ (c.p. (41), below), much in contrast to the cases pictured in Figs. 1, 2 and 3. The leading $\epsilon$-dependence of $P(t)$ can be algebraically determined, in principle, but this was not yet performed due to the complexity of our expansions.

It is interesting to note from the graphs of Figure 4 that the transition probability gets closer to 1 as $\epsilon$ increases. It would seem that for $\epsilon$ large enough, we could have $P(t)>1$. However, our expansions would not converge in this case, since $\epsilon$ would be greater than the radius of convergence. To illustrate this situation, let us consider the trivial case where $\chi_{1}=0$ and $\chi_{2}=2 F_{0} / \omega$ is not an integer. Since now $f(t)=F_{0}=\omega \chi_{2} / 2$, the Schrödinger equation (4) becomes time-independent, with a simple Hamiltonian given by

$$
H_{2}=\epsilon \sigma_{1}+F_{0} \sigma_{3}=\left(\begin{array}{cc}
F_{0} & \epsilon \\
\epsilon & -F_{0}
\end{array}\right) \text {. }
$$

The propagator $U(t)$ can be computed by elementary methods (f.i., by diagonalising the Hamiltonian), leading to

$$
U(t)=\cos \left(\omega_{0} t\right) \hat{1}-\frac{\sin \left(\omega_{0} t\right)}{\omega_{0}}\left(i F_{0} \sigma_{3}+\epsilon \sigma_{1}\right),
$$


where $\omega_{0}:=\sqrt{F_{0}^{2}+\epsilon^{2}}$. Hence,

$$
P(t)=\frac{\epsilon^{2}}{F_{0}^{2}+\epsilon^{2}} \sin ^{2}\left(\omega_{0} t\right) .
$$

Note that $P(t)<1$ for all $\epsilon$ and all $F_{0}$. For $f(t)=F_{0}$, the generalised Riccati equation (8) admits a particular solution given by the constant $g_{0}=-F_{0}+\sqrt{F_{0}^{2}+\epsilon^{2}}$. It was shown in 6 that the $\epsilon$-expansion (15) for $f(t)=F_{0}$ coincides, as expected, with the Taylor expansion (centred at $\epsilon=0$ ) of $g_{0}$. Thus, our method has a clearly restricted region of convergence defined by $|\epsilon|<\left|F_{0}\right|$.

Let us see what these last considerations mean numerically. First we set $F_{0}=0.4$. Figure 5 shows plots of $P(t)$ and $N(t)$ for three critical values of $\epsilon: \epsilon=0.30<F_{0}$, $\epsilon=0.40=F_{0}$ and $\epsilon=0.43>F_{0}$, where our expansions are not supposed to converge. We also show in Figure 5 (dashed lines) plots of $P(t)$ calculated according to (41). Looking at $N(t)$ and at the deviation of the perturbatively computed transition probability from the one calculated via (41), we conclude that the region of convergence of our expansions is restricted to $|\epsilon|<\left|F_{0}\right|$, as expected. It is important to note that when $\epsilon<F_{0}$, our computation of the propagator matches exactly with the non-perturbative solution (40). This can be seen from comparing $P(t)$ calculated perturbatively and nonperturbatively via (41), as shown in Figure 5 .

\section{FINAL REMARKS}

We stress that, at least for the case of monochromatic interactions examined in Section IV, the errors obtained are very small and bounded as time increases (see the behaviour of $N(t)$ in figures of Section [V]). This is due to the absence of secular terms in our perturbative expansions and its uniform convergence in time. As a consequence, one can study the long-time behaviour of the quantum system (3)-(4) in a controlled way.

Another important feature of our method is that it can be numerically implemented with relatively simple computer codes. Indeed, the algorithm described in Section [II] to calculate the unitary propagator $U(t)$ consists, essentially, in simple computations involving the Fourier coefficients of the functions $q$ and $q^{2}$, which are known in a closed form (see (22) and (23)). This is an important advantage against other perturbative approaches, based f.i. on the Dyson expansion (2) which, in general, cannot be evaluated in a simple manner (not to mention the fact that such expansion is not uniform convergent in time, as we have stressed in the introduction of this paper). To sum up, our method is not only mathematically rigorous, but also very useful for practical purposes, where it can be applied with great generality, leading to very accurate results.

\section{ACKNOWLEDGMENTS}

We are grateful to A. Sacchetti for discussions. J. C. A. Barata was partially supported by CNPq. D. A. Cortez was supported by FAPESP.

[1] I. I. Rabi. Phys. Rev. 31, 652-654 (1937).

[2] F. Bloch and A. Siegert. Phys. Rev. 57, 522-527 (1940).

[3] S. H. Autler and C. H. Townes. Phys. Rev. 100, 703-722 (1955).

[4] W. F. Wreszinski. Helv. Phys. Acta 70, 109-123 (1997).

[5] A. Sacchetti. J. Phys. A. Math. Gen. 34 10293-10306 (2001).

[6] J. C. A. Barata. Rev. Math. Phys. 12, 25-64 (2000).

[7] J. C. A. Barata. Ann. Henri Poincaré 2 963-1005 (2001).

[8] J. C. A. Barata and D. A. Cortez. Submitted to publication. math-ph/0201008 v2.

[9] J. C. A. Barata and W. F. Wreszinski. Phys. Rev. Lett. 84, 2112-2115 (2000).

[10] R. P. Feynman, F. L. Vernon Jr. and R. W. Hellwarth. J. Appl. Phys. 28, 49 (1957).

[11] V. G. Bagrov, J. C. A. Barata, D. M. Gitman and W. F. Wreszinski. J. Phys. A. Math. Gen. 34 10869-10879 (2001).

[12] F. Grossmann, T. Dittrich, P. Jung and P. Hänggi. Phys. Rev. Lett. 67, 516-519 (1991). 

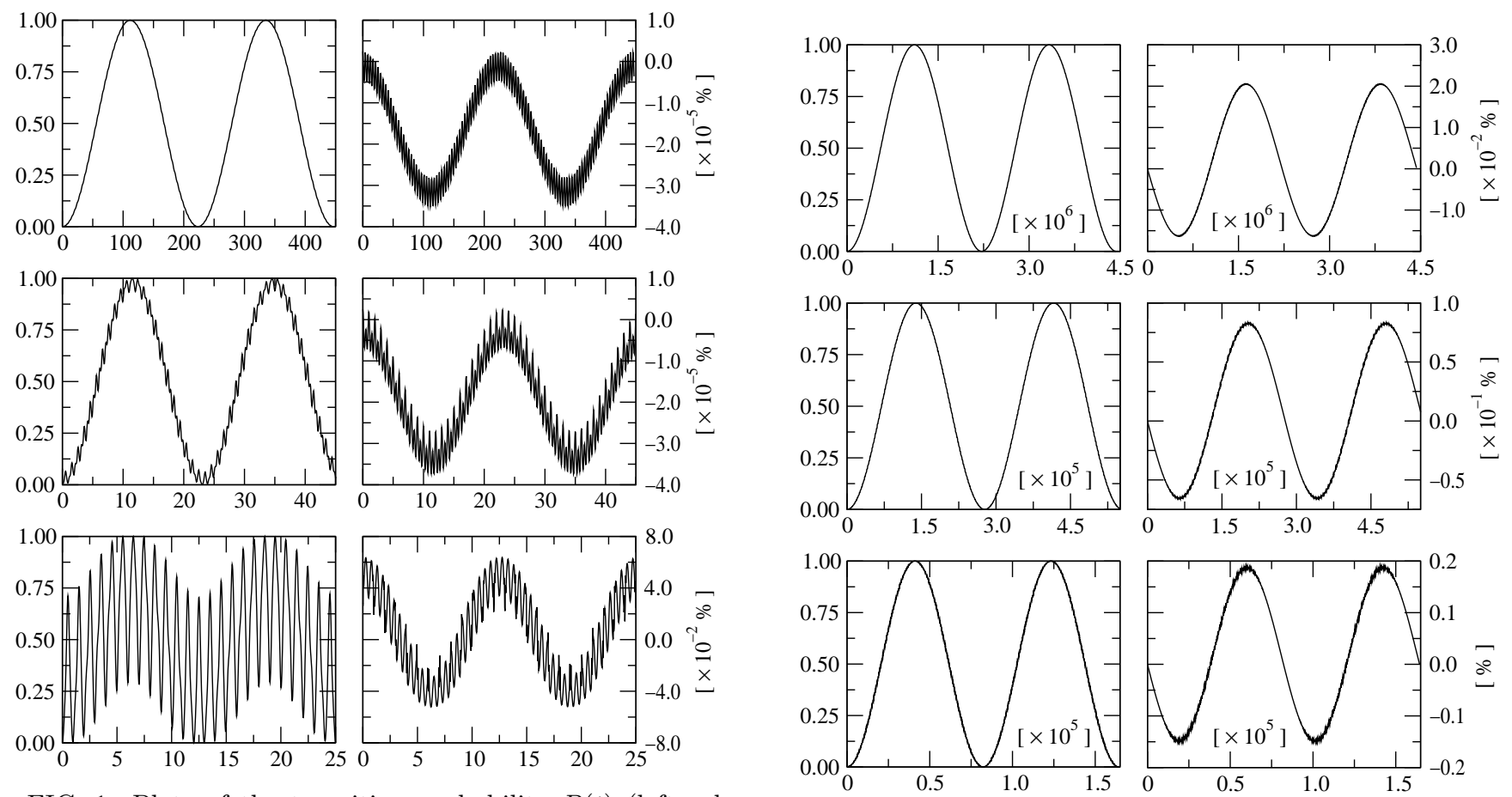

FIG. 1. Plots of the transition probability $P(t)$ (left column) and the deviation from unitarity $N(t)$ (right column) as a function of time (measured in units of $T_{\omega}$ ) for various $\epsilon$. We considered case (A) of Table [1, with $\omega=1.0, \chi_{1}=2 / \omega$ and $\chi_{2}=0$. We used $\epsilon=0.01,0.10,0.40$ in the top, middle and bottom rows, respectively.
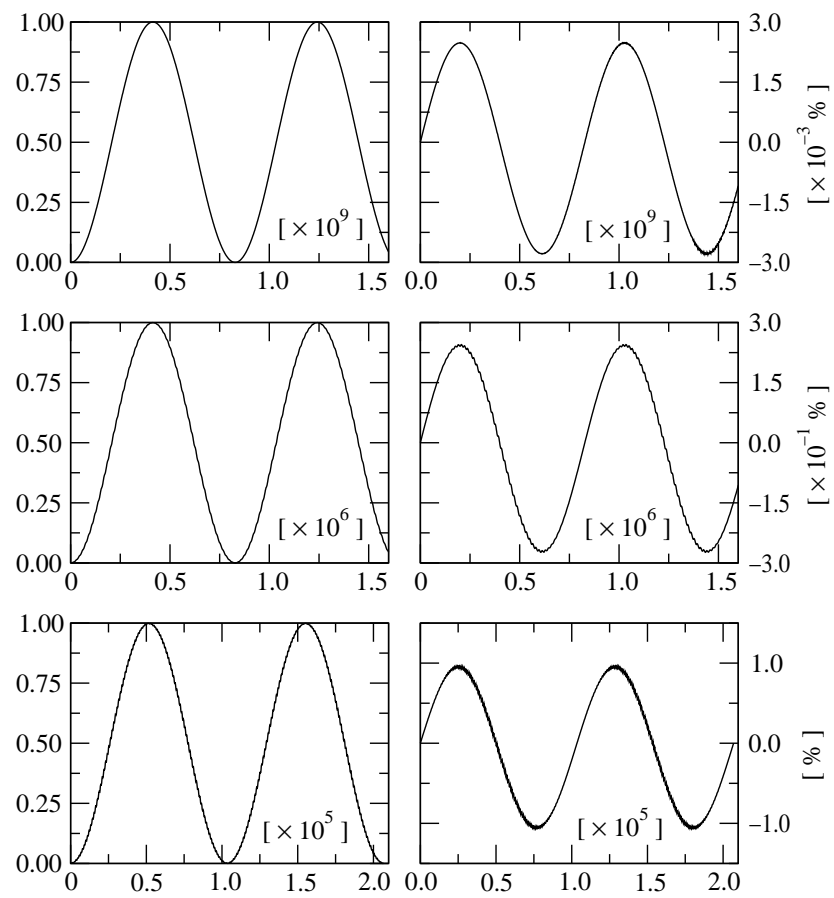

FIG. 2. The same of Figure 1, but we now considered case (B) of Table 1 , with $\omega=10.0, \chi_{1}=x_{1}, x_{1}$ being the first positive zero of $J_{0}$ and $\chi_{2}=0$. We used $\epsilon=0.01,0.10,0.20$ in the top, middle and bottom rows, respectively. Note that the time scale is multiplied by the factor in the square bracket.
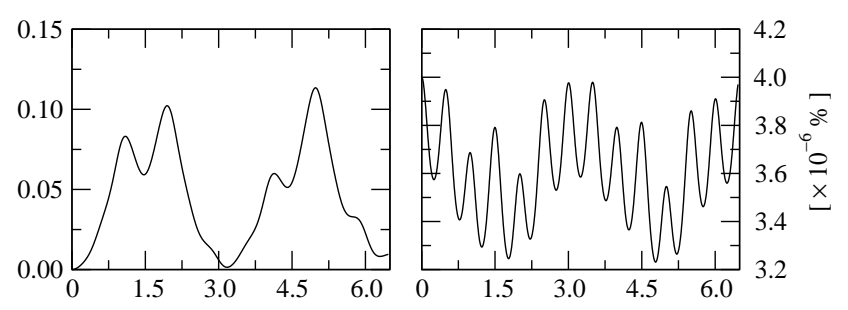

FIG. 3. The same of Figure 2, but now with $\chi_{1}=x_{2}$, $x_{2}$ being the second positive zero of $J_{0}$ and $\chi_{2}=0$. We used $\epsilon=0.10,0.20,0.30$ in the top, middle and bottom rows, respectively.
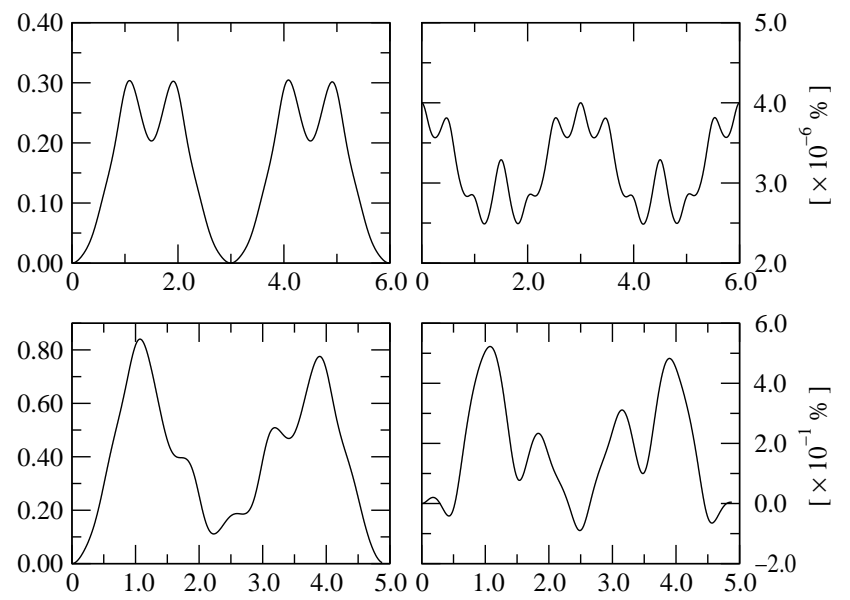

FIG. 4. The same of Figure 1, but we now considered case (C) of Table If, with $\omega=1.0, \chi_{1}=1.0$ and $\chi_{2}=0.3$ (not integer). We used $\epsilon=0.05,0.10,0.20$ in the top, middle and bottom rows, respectively. 

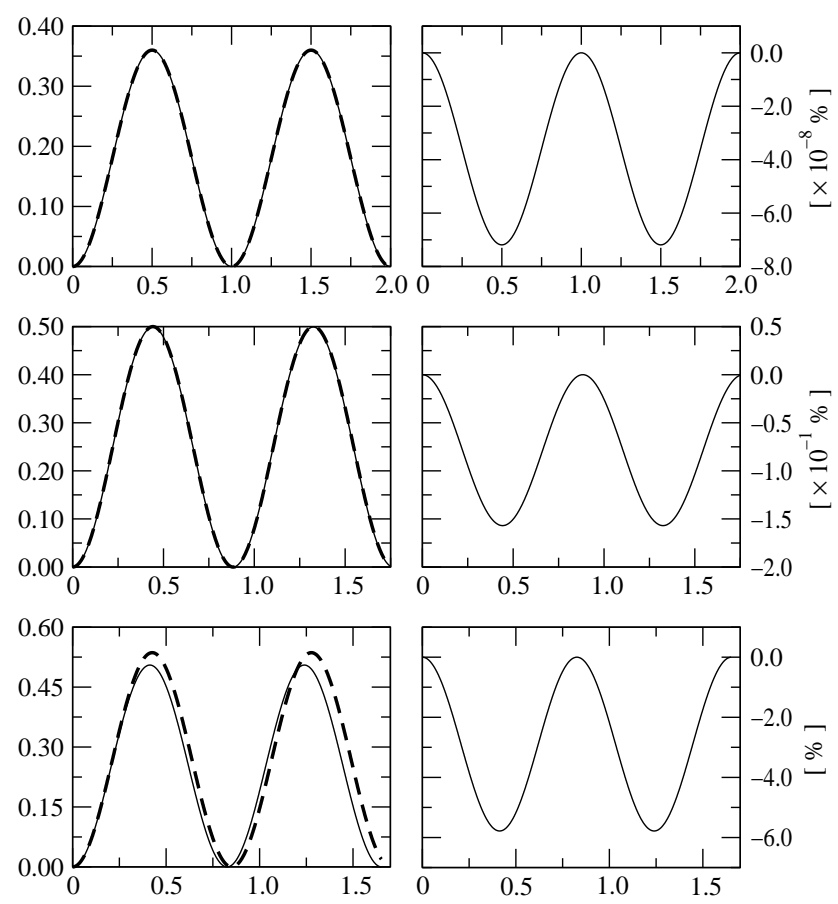

FIG. 5. The same of Figure 1 , but now with $\chi_{1}=0$ and $\chi_{2}=0.8\left(F_{0}=0.4\right)$. We used $\epsilon=0.30,0.40,0.43$ in the top, middle and bottom rows, respectively. The dashed line represents $P(t)$ calculated via formula (41). 